\title{
Innovation Waves, Self-organised Criticality and Technological Convergence.
}

\author{
Rainer Andergassen, Faculty of Economics \\ (Rimini), University of Bologna \\ Franco Nardini, Department of Mathematics for \\ the Social Sciences, University of Bologna \\ Massimo Ricottilli, Department of Economics, \\ University of Bologna \\ Dipartimento di Scienze Economiche, Piazza \\ Scaravilli n. 2, 40126 Bologna, Italy
}

March 27, 2003

\begin{abstract}
The purpose of this paper is to investigate the evolutionary process of imitation and innovation as a process of searching in a given neighbourhood of firms. Networks are the main source of information for firms willing to actively search and upgrade and which define the reachable neighbourhood whose width is strictly related to cognitive distance. We have identified two major forms of information setting off innovative behaviour: the first comes in the shape of random events which are exogenous, at least in terms of the firms' own search activity, while the second is determined by searching for technological opportunities in other economic sectors. It is this activity that generates the spreading of a new technological paradigm and that makes for technological convergence. All firms are a heterogeneous set of agents bounded by their competence, technological specificity and, more generally, rationality. The spreading of information through cognitive neighbourhoods allows firms to gradually acquire full knowledge leading to innovation waves. Imitation follows innovation as firms attempt to glean information on best practise techniques to join their sector technological leaders. Whilst innovators are temporarily allowed to reap quasi rents the imitative band wagon effect drives the profit rate down to its normal level. Productivity growth lowers the prices of sectors involved in the process of technological advance causing obsolescence and, thus, creative destruction in a Schumpeterian sense.
\end{abstract}

JEL classification numbers: D50, L10, O30,

Keywords: Technological change, Self-organized criticality, Innovation and diffusion, Innovation waves, Creative distruction. 


\section{Introduction}

That technical change is a powerful engine of growth is now widely recognized by economic theory, especially the strain which draws inspiration form the Schumpeterian tradition. The dynamic process that technical change sets in motion can appropriately be understood in terms of a virtuous circle weaving actions and feed-backs into a pattern of self supporting and reinforcing events. Technological upgrading supported by either innovation or imitation normally opens up new profit opportunities; other things remaining equal, the expected profit rate to be earned by undertaking technologically improved activities rises bringing about investment demand for new means of production, machinery, plant, tools, instruments and productive services which are meant either to embody new techniques or realize new products. Higher investment then translates into aggregate demand growth enhancing internal resources to conduct innovative search and supporting endogenous learning processes linked to increased activity. This is a self reinforcing process the limit to which is set by the strength of the search process leading to either innovation or imitation and by the waning of the higher profit rates, initially warranted by upgrading, due to bandwagon effects.

Technological change implies 'creative destruction'. The latter can simply be viewed as a process through which innovations are embodied into new capital goods and principles of production which render existing plant obsolete and thus drive the economy towards a wave of scrapping and replacement. This approach can be dealt with in quite different ways. One is the reductionist approach which views 'creative destruction' as merely an equilibrium problem between the contrasting forces of gains to be reaped from profits streaming from innovative activity and the losses incurred from other people's innovative success (Aghion and Howit, 1998). Innovation, according to this view, results from investing some appropriate resource, usually quantities of labour or human capital into specific production processes in competition with employment of the same in manufacturing final goods, as in well known models of the so called new growth theories (e.g. Romer 1990, Lucas 1986). Equilibrium is reached when a steady state is achieved for solutions allocating labour (or available time) between these two branches of activity. The basic message put across by these models is that, in order to depict the impact of technological change, it is expedient to partition the economy in two sectors between which optimally allocate a scarce resource endowment. Innovations then depend on the number of workers employed to produce them given either an exogenous capability and or random innovative or imitative events occurring according to a Poisson arrival rate.

Two issues deserve closer attention. How and why innovation and, indeed, imitation occur is indeed a crucial problem. But while there is a clearly exogenous component in all imitative and innovative processes which is understandably random, technical change is also actively sought by firms and organization, a fact that makes the process of change partly endogenous and a consequence of economic activity itself. The other issue concerns equilibrium. The effort aimed at bringing about change is continuous and no element of the system, least of all 
innovation resources, can be taken as given when the process is largely endogenous and dependent on feedback's. The problem thus lies in giving a satisfactory account of the determinants of firms' imitative and innovative behaviour. Recent economic literature has persuasively argued that efforts directed at seeking ways to upgrade technologically the productive apparatus is a bounded search in a space of opportunities that become visible as the process unfolds. This search is necessarily local and subject to evolution as skills and competence are acquired and learning from experience takes place. Literature belonging to the evolutionary approach (Nelson and Winter, 1982; Dosi, 1988) has persuasively argued that searching is a process of discovery within a bounded neighbourhood. The latter can be defined as the set of technological possibilities determined by a given paradigm which not only binds related techniques but also determines the as yet unknown but reachable new ones given the initial situation, i.e. the extant technique being used or the set of known ones. Formal ways to deal with this process of searching, which is locally random but dynamically situated along a trajectory, have recently been devised (Auerwald et. al, 2000). In this context, techniques are usefully thought of as a recipe of ingredients the modification of which and the new knowledge which is then acquired yield a gradual technological upgrading, measured by higher productivity or lower costs. As mentioned above, this process of searching can in practise be conducted by imitation of techniques in use in the sector of production in which a firm happens to be in activity or through more, full-fledged innovation. The change of a recipe ingredient normally results in an incremental improvement, as in learning by doing processes. Sticking to this culinary parable, an innovation generally entails a radical change in the entire recipe since it often implies a new combination of completely new ingredients. These new combinations may be quite new to the economy: a truly unprecedented innovation which gives rise to a wave of adoptions within the innovative sector and to a path dependent development of new skills and competencies which creates a technological gap with other sectors. Yet, a new paradigm is introduced (Dosi 1988), opening up a new field of opportunities to other sectors conducting their search for technical upgrading. Thus, the latter stand the chance of innovating their own production recipes spreading the paradigm still further and causing technical convergence (Fai and von Tunzelmann, 2001). When imitating, firms observe frontier techniques implemented by other firms producing the same product, or very similar ones, but when innovating, a more complex process takes place. In either case, a dynamic process ensues, of catching up if it is imitation that is occurring, or of steady pushing forward the known frontier if it is innovation that comes to pass. Statistical dynamic patterns can then be fashioned as studied by Iwai (1994, 2000), Franke (2001).

Two as yet non-stated features of the foregoing reasoning must be stressed. The first is that searching is a process cast into agents' bounded rationality. The latter can neither fully scan the entire domain of technological opportunities in theory available in the whole economic system nor can they immediately or instantaneously translate actual observation of better or applicable techniques into adoptable plans to upgrade and invest. What is required is both time- 
consuming and therefore costly informed sorting out of objects worth studying, such as firms which are apparently operating a more productive technique or techniques whose features are at least potentially transferable, and the painstaking collection of information on all the elements making up the actual technical recipe. It is only through appropriate identification and information that useful learning can be accomplished and then action taken. Identifying informative sources and collecting information are uncertain activities which depend on acquired capabilities, established technological prowess, consolidated knowledge. Investment and growth depends, therefore, in a crucial way on how technological knowledge as it is embodied in actual techniques spreads through the system. The dynamics of these processes of diffusion have been analysed in a compelling way by models of self organizing criticality (Bak, Tang and Wiesenfeld,1988).

The purpose of this paper is to investigate the evolutionary process of imitation and innovation as a process of searching in given neighbourhood of firms. In the case of innovation, the relevant neighbourhood is defined by firms' cognitive capabilities, by their ability to learn and elaborate information stemming from sectors of activity other than their own. In the case of imitation, the relevant neighbourhood is made up by firms on the technological frontier which stand to be imitated within the relevant vertically integrated sector : they are the final good producers as well as the suppliers of the elements of the technical recipe, producers of capital goods, providers of services which enter as inputs. It is this network which is the main source of information for firms willing to upgrade by imitation. In any given economic sector, technologically leading firms stand as a standard to be imitated but complete information on the technique in question can effectively be gleaned depending on the richness of the required informational content: a process which demands time and an organizational routine to accomplish full knowledge. Innovation is a different matter. We have identified two major forms: the first comes in the shape of random events which are quite exogenous, at least in terms of firms' own search activity, while the second is determined by searching for innovative opportunities in other economic sectors. It is this activity that generates the spreading of a new technological paradigm and that makes for technological convergence. The paper mainly investigates this second form of innovative activity. Firms search within a neighbourhood of other firms placed within a negotiable technological distance and at the same time, by searching, stand the chance of idiosyncratic shocks. In this sense, all firms are a heterogeneous set of agents bounded by their competence, technological specificity and, more generally, rationality.

Technological progress is implemented through investment in new techniques. The paper generates investment by resorting to a simple function which dictates that all profits are ploughed back in the production process. But investment always occurs in the form of improved methods of production, be it through imitation or through innovation. In order to determine profits and their rate and thus investment, it is necessary to resort to a price system which values outputs and inputs, distributes the net product between wages and profits and is able to describe the dynamics of productivity growth as a consequence of technical advancement. The method devised to catch all these important fea- 
tures of the growing and, more, of the changing economy is that worked out in the simplified but robust model due to Pasinetti (1981). What matters in this context is exclusively direct labour and capital as measured by indirect labour, an approach which neatly highlights distribution and allows to map out the contours of increasing productivity.

The second section of this paper sets the main characteristics of the firms' search process, the third deals with innovation, imitation and technological paradigms and the fourth investigates the properties of the price system and profit rate evolution. Section five, finally, illustrates creative destruction and asymptotic behaviour and section six draws the conclusions.

\section{The search process}

The economy we model is a population of firms $F$ distributed over $J$ vertically integrated sectors producing as many different final goods. There is a fixed number $F_{j}$ of firms in each sector $j, \sum_{j=1}^{J} F_{j}=F$. At each time $t$ firms are distributed as a result of past activity across an ordered list of techniques numbered from the less productive to the frontier one $1,2, \ldots N_{t}^{j}-1, N_{t}^{j}$. It is implied that technological knowledge is not a pure and freely available public good. Thus $\sum_{n=1}^{N_{t}^{j}} f_{t}^{j}(n)=1$, where $f_{t}^{j}(n)$ is the share of firms in sector $j$ using technique $n$. Techniques are ranked in terms of their appearance date: if we denote by $T^{j}(n)$ the date ( $n$-th innovation time) when the $n$-th technique had been introduced in sector $j$, then

$$
0=T^{j}(1)<T^{j}(2)<\ldots<T^{j}\left(N_{t}^{j}\right)
$$

where $N_{t}^{j}$ is the number of techniques adopted according to a time sequence in sector $j$ up to date $t$. More recent techniques are more productive; hence technique $N_{t}^{j}$ is also the best practice in sector $j$ at time $t$. Firms lead an active and costly search to improve their extant techniques by either innovating it or imitating the best practice of their sector. Since innovation is a complex process, in a sense far more than imitation, only few leaders can afford it, whereas all other firms try to imitate them. We assume that in each sector $j$ there is a leader pursuing innovation, and $F_{j}-1$ followers trying to imitate it.

When a sector is involved in an innovation wave, productivity gains occur at a rate which is specific of the sector and depends on the amount of information gathered. Let $\lambda_{j}$ stand for such a rate.

Following the approach outlined in the introductory section, leading firms are also bounded by the knowledge base cumulated through past searching, by the skills they possess, more in general by the organizational and technological capabilities they have developed in time. The search activity they carry out is primarily directed at gathering information on, and learning about, new techniques: it is a search for information which extends as far as the mentioned capabilities allow and it is, therefore, local. A fundamental source of information which firms constantly explore is the set of firms which make up a reachable 
neighbourhood, those which are likely to pass on information on new and more productive techniques.

\subsection{Searching to innovate}

The main focus of this paper lies in the innovations which leading firms endogenously achieve by exploring methods and technological principles already implemented elsewhere in the economy although it is recognized that through research and development efforts entirely new techniques may, rather randomly, be contrived. Information is in any case crucial and must be obtained through firms that possess it and which are placed within a network of viable linkages defining cognitively reachable neighbourhood. Neighbourhood relevant for innovation are determined, therefore, by possessed broad cognitive capabilities. In this case, useful information is passed on by firms ranking at the top of their own sectors' techniques on the somewhat simplifying assumption that only innovators can supply information on a frontier technique and that it is them that must therefore be observed in order to learn and innovate. Since the capability of understanding and processing information coming from a different sector and a different technological context depends on the common knowledge basis, the transmission of such information depends on the strength of this shared knowledge which measures the potential intensity of their interaction and the probability of actually passing on relevant information. Let this measure be defined, in general, by $\epsilon^{i, j} \in[0,1]$ for any two leaders belonging to different sectors $i$ and $j$.

Given these assumptions, it must be stressed that the process of searching is both costly and time consuming. Since firms are capability and rationality bounded, they cannot absorb the information required for technological upgrading in one shot but they must gradually scan their relevant neighbourhood, as defined above, normally according to a search routine. Technological leaders in the various sectors, however, do not always yield the same amount of information: indeed, there are technologically complex innovations whose techniques can be appraised only through a long sequence of informational units, let them be called bits, whilst less complex ones need only short bit sequences to be understood.

The measures of cognitive distance, or proximity, thus defined and empirically observable through a statistical procedure, allow, in turn, a rigorous definition of the cognitive neighbourhood, which we label $N^{i}$, through which innovative information can pass through. Owing to bounded rationality we are going to consider an economy where firms are clustered into neighbourhoods with cardinality $S^{*}$. We further assume symmetry between firms $i, j$ in the sense that $\epsilon^{i, j}=\epsilon^{j, i}$. There are, therefore, $\frac{J(J-1)}{2}$ couplings which compose the set:

Definition $1 E=\left(\epsilon^{i, j} \mid i, j=1,2 \ldots J\right) ; \quad|E|=\frac{J(J-1)}{2}$

Given the set $E$, we may now further consider all the neighbourhoods that 
each firm enters with a given number of possible neighbours. Furthermore, since we consider cognitive symmetry between firms we are also led to assume that if a firm is in another's neighbourhood the opposite also applies.

Definition 2 We define a neighbourhood configuration $k$ as the set

$$
\begin{gathered}
\gamma^{k}=\left\{\gamma^{1, k}, \gamma^{2, k}, \ldots \ldots, \gamma^{J, k}\right\} \\
\text { where } \gamma^{i, k} \subset\{1,2 \ldots J\}-\{i\} ;\left|\gamma^{i, k}\right|=S^{*} \text { and } i \in \gamma^{j, k} \Leftrightarrow j \in \gamma^{i, k}
\end{gathered}
$$

These definitions provide a map of cognitive neighbourhoods . The sets of neighbours in each $\gamma^{k}$ are of varying informative value for the firm on account of the cognitive heterogeneity of its members. It follows that a ranking of these neighbourhood can be compiled on the grounds of how enabling they are from the point of view of their informative content, given the combination of probabilities $\epsilon^{i, j}$. A convenient measure of such informative content and of the ease with which information percolates through to let the firm learn and cumulate knowledge for innovation is Shannon's entropy measure (Klir and Folger, 1988). We consider the average entropy as a standard case for the whole economy

$$
M(k)=-\frac{1}{J} \sum_{j=1}^{J} \sum_{i \in \gamma^{j, k}} \epsilon^{i, j} \log _{2}\left(\epsilon^{i, j}\right)
$$

Given $M(k) \in[0, \infty]$ for all $k$ 's, it is possible to compute the minimum, identifying the standard case of a neighbourhood which is most capable of carrying innovative information for the economy as a whole.

Definition $3 N^{i}$ is the neighbourhood of firm $i$ which is, on average, most likely to provide significant innovative information and which is, therefore, the cognitively relevant neighbourhood for the diffusion of innovative technologies.

$$
N^{i}=\gamma^{i, \bar{k}}
$$

and

$$
\bar{k}=\arg \min M(k)
$$

This definition allows us to identify an innovator's standard neighbours in the lattice $L$ for all innovating firms. We consider the average probability, within the neighbourhood of firm $i$, of passing information on $\hat{\epsilon}^{i}=\frac{1}{\left|N^{i}\right|} \sum_{j \in N^{i}} \epsilon^{i, j}$. The property that the neighbourhood structure is of minimum entropy together with the assumption that $J$ is very large, implies that $\hat{\epsilon}^{i} \approx \hat{\epsilon}^{j}$, for each leader $i, j$. Thus, for the following we consider the average probability of passing information on, $\hat{\epsilon}=\frac{1}{J} \sum_{i=1}^{J} \hat{\epsilon}^{i}$, where $\lim _{J \rightarrow \infty} \frac{1}{J^{2}} \sum_{i=1}^{J}\left(\hat{\epsilon}^{i}\right)^{2}=0$.

The probabilities $\hat{\epsilon}$, measuring cognitive relationships, depend in each neighbourhood on the number of firms which are nested therein. This proposition 
follows directly from the very definition of neighbourhood as the locus of dense interfirm externalities. Economic history and studies in the Marshallian tradition have provided plenty of evidence for this fact. In particular, literature on industrial districts indicates that firms tend to cluster according to a predictable pattern often determined by agglomeration economies based on shared knowledge and know how. The greater is the number of firms in any given cluster , the greater is the cognitive correlation and the greater the probability that information spread across the cluster. From the point of view of the economy as a whole, firms' cognitive capability is enhanced by specialization and division of labour. The deeper they are, the greater is the scope for learning on the one hand and the more firms are systematically linked to other firms with differing but related knowledge, on the other. Cognitive capabilities and relationships are, therefore, greater where economic development has widened sectorial specialization, where the number of sectors is greater and denser, each one of them with its own technological leadership, providing greater scope for learning and requiring firms to possess a large variety of technical capabilities bridging the distance between sectors.

These considerations support the following assumption.

Proposition 4 The average probability for the economy as a whole that information be passed on is

$$
\hat{\epsilon}=1-\alpha \frac{1}{J}
$$

$\alpha$ can be interpreted as the threshold of specialization, measured by the number of sectors, such that no meaningful and relevant information spills through: $J^{*}=\alpha$. It is easy to check that $\hat{\epsilon}=1$ is the asymptote for $J \rightarrow \infty$. These assumptions and definitions reduce the normally high empirical heterogeneity of the various firms in the economy. Since the interest of this paper lies primarily on the average capability of the entire economy to engender innovation endogenously through intersectoral diffusion of new technological principles, we shall normally refer to $\hat{\epsilon}$ as the average probability that information gets through, $\alpha$ being the critical number of sectors.

A leading firm's neighbourhood is susceptible of providing information on technologies of differing complexity requiring bit sequences of appropriate length. Thus, in any $N^{i}$ there is likely to be information on technologies requiring both long and short sequences provided either exogenously or by $S^{*}$ neighbours. Which of these sequences a given innovator locks into is largely a matter of probability, given the same propensity to invest in the innovation process. The probability measure is given by the proportions of sectors which make up the economy. The more technologically advanced are the sectors present in a given economy, and thus in a given neighbourhood, the greater is the probability that such sectors be observed to set off a long sequence of bit collection requiring many observations of leading firms within the neighbourhood ${ }^{1}$.

\footnotetext{
${ }^{1}$ The process leading to a given sector configuration can be thought of as a self reinforcing
} 
We summarize the above mentioned facts into the following

Axiom 5 For each innovation, the generic innovator $i$ stands probability $p_{z}$ of locking into a technological search $z$, requiring an informative sequence of $S_{z}$ bits, $z=1,2, \ldots, Z$. Obviously $\sum_{z=1}^{Z} p_{z}=1$.

Without loss of generality we rank these possible technological searches as

$$
S_{1}<S_{2}<\ldots<S_{Z}
$$

It is a fairly well established fact that, at least on average, innovations requiring a greater effort, which is here measured by the number of bits collected, allow for larger productivity gains, a fact that, together with (1), leads us to the following assumption:

Axiom 6 Let us denoted by $\lambda_{z}$ the productivity growth rate resulting from the innovation produced by a search of type $z$, then

$$
\lambda_{1}<\lambda_{2}<\ldots<\lambda_{z}
$$

Since information is mainly retrieved from neighbours, we are led to assume that the longer are the informative sequences, the larger is the number of neighbours $S^{*}$, which each leader contacts in its search. We formalize this fact in the following

Axiom 7 The number of neighbours is equal to the mean value of the random variable length of the informative sequence

$$
S^{*}=\sum_{z=1}^{Z} p_{z} S_{z}
$$

\section{The innovation process}

The richly heterogeneous context outlined above provides the backdrop on which to discuss innovation. It highlights the fundamental fact that firms cluster in knowledge specific neighbourhood. Relationships within such aggregates are crucial for firms' operative success and for their search effort to apply technical progress (Potts 2001). The assumptions made above allow us to deal with the characteristics of the process of innovation as it emerges from information diffusing through the system beginning with a single exogenous event which

mechanism which locks an economy to develop technologies of a given complexity. (See Arthur, 1994) 
idiosyncratically arrives into the system. This information is acquired by a firm to initiate a learning process or to complete it if it already possesses enough information to develop it into a new technology and investment.

Some simplifications must now be introduced since we wish to develop a fairly detailed yet mathematically tractable description of this innovation process. To do this we adopt a mean field solution to the problem. This approach is justified since, although embedded in a specific context, firms learn about novel technologies and gather knowledge by means of information that 'travels' through neighbourhood of least entropy. It is the latter that matter for diffusion. Hence, the degree of heterogeneity is, from the point of view of the learning process, lessened since cognitive difference is restricted to where it is actually relevant. Average entropy is one of the economy's structural characteristics: technological diffusion is clearly harder where it is higher. Furthermore, firms acquire information through exposure to other firms which have already collected all the necessary informative material through a gradual process and upon the completion of which they invest. This process of osmosis concerns all leaders, albeit within the boundary of cognitive neighbourhood, but it is subject to the probability of encountering a fully informed and ready to invest firm given the probability that information be passed on. In principle, the probability of being in a specific state of information completeness, or learning status, depends on a complex web of correlations between the states of firms in the neighbourhood and their probabilities of being there. Furthermore, the transitional probabilities of moving from one state to the next depend on such probabilities and on the other firms' transitional probabilities.

We propose a mean field approximation which ignores such complex correlations and de-couples the probabilities of the $J$ innovators ${ }^{2}$. Accordingly, we analyse innovators' average densities in the various states of informative completion independently of the sector they belong to while proposition 4 sets the average probability of information to be actually transferred. The fact that information transits through least entropy neighbourhood reduces the error in estimating the true probability of firms to actually learn and upgrade. Firms remain heterogeneous regarding their cognitive bases, the technology in use, the sector they belong to and the informational content of their innovations. As argued above, the state of informative completion depends on the technological sequence leading firms on the way to innovation lock into.

The stationary distribution represents in this case the probability, in the stationary state, that a given firm has accumulated a certain number of bits of information. As to the question of the probability that such information does get across, i.e. the strength of the relationship between elements, the minimum entropy neighbourhood structure together with the assumption that $J$ is very large guarantees that the average probability $\hat{\epsilon}$ captures the essence of the information spill-over dynamics. This average probability is to be distinguished from the average probability that information be able to travel across distant

\footnotetext{
${ }^{2}$ Vespignani and Zapperi (1998) provide evidence through computer simulations that the mean-field approach describes well the stationary state behaviour of the model.
} 
neighbourhood, i.e. that cognitive linkages be actually established over very far clusters. We accommodate this less likely event without major complications. Let this probability be designated by $\tilde{\epsilon}$.

As a consequence of these assumptions, all leaders in the system may be ranked in terms of the information currently possessed. If $J \rho_{s}(t)$ denotes the number of firms possessing $s$ bits of information, then there are $J \rho_{a}(t)$ firms which have completed the collection of information and are in a position to introduce an innovation at time $t$. We refer to the $J \rho_{a}(t)$ firms innovating at time $t$ as to firms in the active state; we say that a firm is in the critical state if it needs only one bit of information to become active. At time $t$ there are $J \rho_{c}(t)$ such firms. Remaining firms are at various stages of incomplete information: $J \rho_{s}(t)$. After each innovation, firms begin a new cycle of search; there are $J \rho_{0}(t)$ firms in this phase at time $t$.

Summing up, information may be acquired in three different ways. First, when firms introduce an innovation, being in state $a$,all their neighbours get a chance to observe it with probability $\hat{\epsilon} \rho_{a}$. Hence the fraction of leaders which probably become able to change over from a critical to an active state is $S^{*} \hat{\epsilon} \rho_{a} \rho_{c}$. Secondly, a firm may occasionally be contacted outside the relevant neighbourhood, bridging two network clusters, with probability $\tilde{h}$. Information is then obtained with probability $\tilde{h} \tilde{\epsilon}$ and the fraction of critical state firms able to upgrade to an active state through this avenue is $\tilde{h} \tilde{\epsilon} \rho_{c}$. Thirdly, we also consider the case of an entirely exogenous innovative event with a Poisson arrival rate of $\bar{h}$, the implication being that the fraction of firms reaching the active state is, in this third case, $\bar{h} \rho_{c}$.

In order to simplify the exposition we consider, for the following, the case of only two informational sequences, $S_{2}>S_{1}$. Further, we assume that the fraction of leaders engaged in collecting $S_{1}$ informational bit is $p$, while $1-p$ is the fraction of those collecting $S_{2}$ bits of information. Consequently, $S^{*}=p S_{1}+(1-p) S_{2}$. We define state $k \equiv S_{1}-2$. In Figure 1 we depict the state space dynamics in the case where $S_{1}=3$ and $S_{2}=7$.

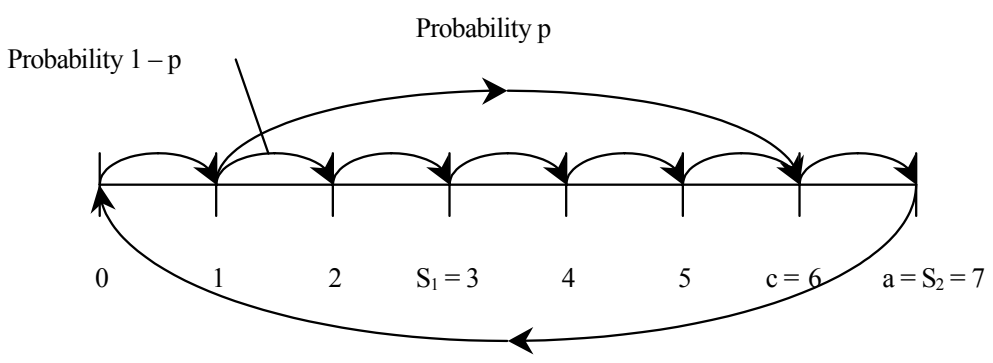

Figure 1: The state space dynamics in the case of $S_{1}=3$ and $S_{2}=7$. 
This process of accumulation of information may formally be described by the following differential equations for the shares $\rho_{s}$, for $s=0,1, \ldots, c, a$.

$$
\begin{gathered}
\dot{\rho}_{a}=\left(h+S^{*} \hat{\epsilon} \rho_{a}\right) \rho_{c}-\rho_{a} \\
\dot{\rho}_{c}=-\left(h+S^{*} \hat{\epsilon} \rho_{a}\right) \rho_{c}+\left(h+S^{*} \hat{\epsilon} \rho_{a}\right) \rho_{c-1}+p\left(h+S^{*} \hat{\epsilon} \rho_{a}\right) \rho_{k} \\
\dot{\rho}_{c-1}=-\left(h+S^{*} \hat{\epsilon} \rho_{a}\right) \rho_{c-1}+\left(h+S^{*} \hat{\epsilon} \rho_{a}\right) \rho_{c-2} \\
\cdots \\
\dot{\rho}_{k+1}=-\left(h+S^{*} \hat{\epsilon} \rho_{a}\right) \rho_{k+1}+(1-p)\left(h+S^{*} \hat{\epsilon} \rho_{a}\right) \rho_{k} \\
\dot{\rho}_{k}=-\left(h+S^{*} \hat{\epsilon} \rho_{a}\right) \rho_{k}+\left(h+S^{*} \hat{\epsilon} \rho_{a}\right) \rho_{k-1} \\
\cdots \\
\dot{\rho}_{0}=-\left(h+S^{*} \hat{\epsilon} \rho_{a}\right) \rho_{0}+\rho_{a}
\end{gathered}
$$

where $h=\bar{h}+\widetilde{h} \widetilde{\varepsilon}$. The normalization condition requires that

$$
\rho_{a}+\rho_{c}+\ldots+\rho_{0}=1
$$

In order to underlie the impact of mere diffusion percolating through close cognitive neighbourhood and isolate it from rarer occurrences, namely information passing over distant clusters or mere exogenous events, we assume both $\bar{h}$ and $\widetilde{h}$ to be very small; we are, therefore, interested in first order expansions as $h=\bar{h}+\widetilde{h} \widetilde{\varepsilon} \longrightarrow 0$. In the stationary state we have that

$$
\begin{gathered}
\rho_{c}=\rho_{k}=\rho_{k-1}=\ldots=\rho_{0} \\
\rho_{S_{2}-2}=\rho_{S_{2}-3}=\ldots=\rho_{k+1}=(1-p) \rho_{k}
\end{gathered}
$$

where

$$
\rho_{c}=\frac{1}{S^{*}}
$$

The solutions above describe a state in which the system settles for given exogenous shocks compacted into $h$ as a result of the interplay of two different sources. The first is exogenous, supplying a new 'bit' of information, with probability $h$, to the firm's current technological knowledge either from institutions outside the strictly defined economic system or from other firms situated very far away in the network of connected neighbourhood. The second is endogenous, providing information from a member of the firm's neighbourhood which is itself in the process of innovating. The former, therefore, is the exogenous dynamics whilst the latter represents the endogenous propagation of a disturbance. If this system received no exogenous shocks, there would be no innovation process. We wish to show that even in the case of rare exogenous shocks, the economy we have depicted is subject to innovative processes by diffusion of technological principles from one sector to its neighbours causing technological convergence. Thus, when a rare exogenous shock hits a single leader in a particular sector, a chain of subsequent innovations is triggered spreading to the whole economy.

\subsection{The imitation process}

As observed above imitation is not only in some sense simpler than innovation, but it is also a basically different task. The frontier technique, which is aimed at 
by imitators, is already there and imitators have to detect how this new recipe combines a possibly large number of ingredients into a new, may be complex, combination. It is clear that the larger is this number, the larger is the number of possible unsuccessful trials, and the longer is the expected time for a successful imitation.

Thus, it is natural to assume that successful imitations appear as a Poisson random arrival process and the arrival rate decreases as the informational content of the innovation increases; the more complex the innovation is, the harder is its imitation. On the other hand it is clear that imitation in a sector becomes easier and easier the more firms has succeeded in adopting the best practice in this sector. These two effects may be formalized into the following assumption (see also Iwai p. 171).

Axiom 8 The probability that an imitator succeeds in adopting the best practice in sector $j$ during the small time interval $d t$ equals $\mu\left(S_{j}\right) F_{j} f_{t}^{j}\left(N_{t}^{j}\right) d t$, where $F_{j} f_{t}^{j}\left(N_{t}^{j}\right)$ is the number of firms currently using the best practice in sector $j$ and $\mu\left(S_{j}\left(N_{t}^{j}\right)\right)$ is a decreasing function of the length ${ }^{3} S_{j}\left(N_{t}^{j}\right)$ of the search that has produced the $N_{t}^{j}$-th innovation in sector $j$..

Since it is clear that the probability that an imitator manages to guess the right recipe of the best practice is much larger that the probability that an innovator has access to a relevant piece of information from outside the system in order to discover a new production technique, we are led to assume that

$$
\mu\left(S_{j}\left(N_{t}^{j}\right)\right)>>h
$$

When an innovation wave reaches sector $j$ a new technique is adopted by the leader, while followers are left behind in the ranking of techniques. If $f_{t}^{j}(n)$ denotes the share of firms in sector $j$ that use technique $n$ at time $t$, then

$$
\begin{aligned}
f_{T^{j}\left(N_{t}^{j}\right)}^{j}\left(N_{t}^{j}\right) & =\frac{1}{F_{j}} \\
f_{T^{j}\left(N_{t}^{j}\right)}^{j}\left(N_{t}^{j}-1\right) & =\lim _{t \rightarrow T^{j}\left(N_{t}^{j}\right)-} f_{t}^{j}\left(N_{t}^{j}-1\right)-\frac{1}{F_{j}} \\
f_{T^{j}\left(N_{t}^{j}\right)}^{j}(n) & =\lim _{t \rightarrow T^{j}\left(N_{t}^{j}\right)-} f_{t}^{j}(n) \text { for } n<N_{t}^{j}-1 .
\end{aligned}
$$

Soon after the imitation process begins and leads to a progressive catching up by imitators.

Following Iwai, if the number $F_{j}$ is sufficiently large, the evolution of the shares $f_{t}^{j}(n)$ after time $T^{j}\left(N_{t}^{j}\right)$ can be approximated by continuous functions

\footnotetext{
${ }^{3}$ We refer to $S_{j}\left(N_{t}^{j}\right)$ also as to the information content of the $N_{t}^{j}$-th innovation.
} 
of $t$

$$
\begin{aligned}
f_{t}^{j}\left(N_{t}^{j}\right) & =\frac{1}{1+\left(F_{j}-1\right) e^{-\mu\left(S_{j}\left(N_{t}^{j}\right)\right) F_{j}\left(t-T^{j}\left(N_{t}^{j}\right)\right)}} \text { for } t>T^{j}\left(N_{t}^{j}\right) \\
f_{t}^{j}(n) & =\frac{f_{T^{j}\left(N_{t}^{j}\right)}^{j}(n)\left(1-f_{t}^{j}\left(N_{t}^{j}\right)\right)}{1-f_{T^{j}\left(N_{t}^{j}\right)}^{j}\left(N_{t}^{j}\right)} \text { for } n<N_{t}^{j} \text { and } t>T^{j}\left(N_{t}^{j}\right)
\end{aligned}
$$

By (16) it is plain that in most cases when a further innovation is introduced in sector $j$ most of the $F_{j}$ firms have completed the catching up process and are using the best practice.

\subsection{Technological waves and technological paradigms}

If a firm becomes active because of the arrival of exogenous information at time $T$, we denote by $V_{T}$ the number of firms upgrading their productive techniques soon after $T$. Then the following result holds.

Theorem 9 In the limit for $h \rightarrow 0$ the expected value of $V_{T}$ is

$$
E\left(V_{T}\right)=\left.\frac{\partial}{\partial h} \rho_{a}\right|_{h=0}=\frac{1}{(1-\hat{\epsilon}) S^{*}}
$$

The proof can be found in Vespignani and Zapperi (see appendix A).

This is an interesting result since it states that, given a situation in which equilibrium prevails for a very weak, approximately zero, exogenous force $(h \rightarrow$ 0 ), if the latter is allowed to increase, an idiosyncratic shock hits a single firm, then an innovation wave ensues ${ }^{4}$. This is so given Proposition 4

$$
E\left(V_{T}\right)=J \frac{1}{\alpha S^{*}}
$$

The expected number of firms concerned is a fraction of the total $J$ given the factor $\frac{1}{\alpha S^{*}}$. It is interesting to note that the wave size depends, given $J$, on parameters $\alpha$ and $S^{*}$ : the larger is the former, meaning the larger is the number of sectors required to generate positive interaction, the lower is the number of firms likely to be involved; the same occurs the larger is the number of neighbours. We refer to (8) as to the expected size of the innovation wave.

We denote by $N_{t}$ the number of innovation waves occurred from time 0 to time $t$. We call waiting time for the $N_{t}+1$ innovation the time $T\left(N_{t}+1\right)-T\left(N_{t}\right)$ separating two successive innovations. The following result holds.

Theorem 10 In the limit for $h \rightarrow 0$ the expected waiting time $\omega$ is

$$
\omega=E\left(T\left(N_{t}+1\right)-T\left(N_{t}\right)\right)=\frac{S^{*}}{h}+o\left(\frac{1}{h}\right) \text { for } h \rightarrow 0 .
$$

\footnotetext{
${ }^{4}$ Borrowing from the mean-field analysis of sandpile models, we call "avalanche" the set of firms involved; $V_{T}$ is the avalanche size.
} 
Proof. First we calculate the probability distribution

$$
W(s)=\operatorname{Pr}\left\{T\left(N_{t}+1\right)-T\left(N_{t}\right) \leq s\right\} .
$$

It is clear that

$$
d W(s)=(1-W(s)) h \rho_{c}\left(N_{t}+s\right) d s .
$$

Integrating (11) we obtain

$$
W(s)=1-e^{-h \int_{0}^{s} \rho_{c}\left(N_{t}+u\right) d u}
$$

Now (12) may be directly inserted into the mean value formula and integrating by parts

$$
\omega=\int_{0}^{+\infty} s d W(s)=\int_{0}^{+\infty} e^{-h \int_{0}^{s} \rho_{c}\left(N_{t}+u\right) d u} d s
$$

Having in mind (7), the limit of (13) is precisely (9).

Since in our framework and in our first order approximation for $h \rightarrow 0$ the arrival of innovation waves turns out to be a renewal process, the renewal theorem $^{5}$ says that the expected number of innovation waves up to time $t$ will approach asymptotically the ratio between $t$ and the expected waiting time $\omega$, as $t$ becomes very large

$$
E\left(N_{t}\right) \sim \frac{t}{\omega} \text { as } t \rightarrow+\infty
$$

On the other hand $E\left(N_{t}^{j}\right)=E\left(N_{t}\right) E\left(V_{T}\right) \frac{1}{J}$. By theorems 9, 10 and (14) (9) in our first order approximation as $h \rightarrow 0$, the asymptotic behaviour of the expected number of innovation occurred in sector $j$ before time $t$ is

$$
E\left(N_{t}^{j}\right) \sim \frac{h}{\alpha S^{* 2}} t \text { as } t \rightarrow+\infty
$$

Thus, the expected waiting time for the first successful imitation is by far shorter than the expected waiting time (9) of the following innovation wave

$$
\frac{1}{\mu\left(S_{j}\left(N_{t}^{j}\right)\right)}<<\omega=\frac{S^{*}}{h} \quad, \forall j .
$$

\footnotetext{
${ }^{5}$ See Feller (1971) p. 360.
} 


\section{Profit rate, wage rate and relative prices}

We now wish to deal with the impact that innovation and imitation have on profit rates which are, in turn, the key to investment demand. To proceed with a suitable and useful calculation of these rates in the various sectors and relatively to the various techniques, it is expedient to classify sectors by following the approach proposed by Pasinetti $(1981,1993)$, namely by viewing the economy as a set of vertically integrated sectors referred to final goods. This is a simplification but it serves the purpose of concentrating the analysis on the basic determinants of both technical progress and cost of means of production. The advantage is twofold. First, technical progress through innovation can take place in any, sometimes in all, the linkages tying users to producers. Through these linkages, which are also of crucial importance for the spread of information leading to both innovation and imitation, technological improvement yields benefits which percolate forward as costs are lowered and performance enhanced. Thus, final industries are recipient of technological improvement directly through their own search and indirectly through the effort of firms supplying means of production and intermediate goods. Secondly, cost and price are reduced to their most important determinant: integrated labour and wage cost.

We accordingly assume, retaining similar symbols used by Pasinetti (1981), that one unit of output of a final good $j$, produced with technique $n$, requires inputs of direct labour, $a_{j, n}$, a fraction of the duration $m$ of some units of a vertically integrated capital good which is in turn produced by direct labour, $a_{k_{j}, n}$. The production period is unitary and wages are paid at the end of the period; production period and productive life of capital are assumed uniform across sectors and independent of the productive technique; more general assumptions do not yield any interesting generalization, but they entail a considerable complication of the notation.

In what follows, capital capacity will be modelled as being the output of direct labour alone although a more general model can easily be devised to accommodate capital inputs. Firms earn a profit rate, $r_{j, n}(t)$, on the stock of capital required by output, given current prices, $p_{j}(t)$ for final goods and $p_{k_{j}}(t)$ for capital goods, the wage rate $w(t)$, and the productive technique.

As it can be gleaned by these assumptions, the production structure of the economy is simple but it still captures its basic features. Commodities are produced by means of commodities and labour.

The price system we model below is basically a long run equilibrium system, which, however, allows for imbalances and monopolistic quasi rents for innovators in the short run after each innovation wave. When a new and more productive technique is introduced as a consequence of an innovation wave, prices are not affected until the imitation starts, since imitation requires time, innovators can reap the entire extra profit generated by the technical advance for a significant time span. After this, successful imitators generate a flow of investment such as to level out profits for all firms no the technological frontier on each sector.

We denote by $\lambda_{j}(n)$ the productivity growth rate achieved by the leader 
introducing the $n$-th innovation in sector $j$, thus ${ }^{6}$

$$
\begin{aligned}
a_{j, N_{t}^{j}} & =a_{j, N_{t}^{j}-1} e^{-\lambda_{j}\left(N_{t}^{j}\right)}, \\
a_{k_{j}, N_{t}^{j}} & =a_{k_{j}, N_{t}^{j}-1} e^{-\lambda_{j}\left(N_{t}^{j}\right)} .
\end{aligned}
$$

According to axiom 6, the larger is the informative sequence $S_{j}(n)$ giving rise to the $n$-th upgrading, the larger is the resulting productivity growth rate $\lambda_{j}(n)$.

Following Pasinetti, the price of a unit of capital embodying the best technique $N_{t}^{j}$ in sector $j$ at time $t$ is

$$
p_{k_{j}}(t)=a_{k_{j}, N_{t}^{j}} w .
$$

On the other hand it generates a flow of net gains $p_{j}(\tau)-w a_{j /, N_{t}^{j}}$ in its entire productive life $\tau \in[t+1, t+1+m]$, where $p_{j}(\tau)$ is the unit price of commodity $j$ at time $t$. If $r_{j, N_{t}^{j}}(\tau)$ is the rate of profit generated by the above mentioned unit of capital in the same interval $\tau \in[t+1, t+1+m]$, then

$$
p_{k_{j}}(t)=\int_{t+1}^{t+1+m}\left(p_{j}(\tau)-w a_{j, N_{t}^{j}}\right) e^{-r_{j, N_{t}^{j}}(\tau)(\tau-t)} d \tau
$$

If $H^{j}(n)$ is the time separating the $n$-th innovation from the first successful imitation in sector $j$, we assume that

$$
\begin{gathered}
p_{j}(t)=\begin{array}{c}
p_{j, N_{t}^{j}-1} \text { if } t<T^{j}\left(N_{t}^{j}\right)+H^{j}\left(N_{t}^{j}\right) \\
p_{j, N_{t}^{j}} \text { if } t>T^{j}\left(N_{t}^{j}\right)+H^{j}\left(N_{t}^{j}\right) \\
\text { and }
\end{array} \\
p_{k_{j}}(t)=\begin{array}{c}
p_{k_{j}, N_{t}^{j}-1} \text { if } t<T^{j}\left(N_{t}^{j}\right)+H^{j}\left(N_{t}^{j}\right) \\
p_{k_{j}, N_{t}^{j}} \text { if } t>T^{j}\left(N_{t}^{j}\right)+H^{j}\left(N_{t}^{j}\right)
\end{array}
\end{gathered}
$$

Here $p_{j, N_{t}^{j}}$ and $p_{k_{j}, N_{t}^{j}}$ denotes the long run equilibrium prices, which leave out profit rates in all firms on the technological frontier in each sector. Economic history highlights what has come to be taken as a stylized fact: over a fairly long period of time, the tendency of the profit rate is to remain fairly constant

\footnotetext{
${ }^{6}$ It is perfectly possible to assume two different growth rates in the two vertically integrated sectors $j$ and $k_{j}$

$$
a_{j, N_{t}^{j}}=a_{j, 0} e^{-\lambda_{j} N_{t}^{j}}
$$$$
a_{k_{j}, N_{t}^{j}}=a_{k_{j}, 0} e^{-\lambda_{k_{j}} N_{t}^{j}}
$$

however this more general assumption does not yields more general results, but only considerable additional troubles in the notation.

We choose (17) for the sake of simplicity.
} 
together with the labour and profit shares in aggregate net output. This equilibrium may be attained or at least approached only if the productive life of capital $m$ is significantly shorter than the mean waiting time separating two successive innovations in the same sector, a perfectly natural assumption in our context. In these assumptions equation (19) becomes

$$
\frac{p_{k_{j}, N_{t}^{j}}}{w}=\left(\frac{p_{j, N_{t}^{j}}}{w}-a_{j, N_{t}^{j}}\right) e^{-r} \frac{1-e^{-r m}}{r}
$$

Equation (21) is, thus, the efficiency curve of technique $N_{t}^{j}$ highlighting the trade off between profit and wage rates. This implies that the benefits of technical progress are shared out fairly equally by means of a rising real wage rate.

From the previous equations (21) and (18), we can solve for

$$
\frac{p_{j, N_{t}^{j}}}{w}=\left(a_{j, N_{t}^{j}}+\frac{a_{k_{j}, N_{t}^{j}}}{e^{-r} \frac{1-e^{-r m}}{r}}\right)=e^{-\lambda_{j}\left(N_{t}^{j}\right)} \frac{p_{j, N_{t}^{j}-1}}{w} .
$$

Formula (22) clearly shows that increases in productivity which take place in the innovative sectors yield benefits which spread to other sectors not directly concerned through changes in relative prices.

We now pass to the first period after the $N$-th innovation in sector $j$, when the innovator expects extra profits because of his monopolistic power. This period starts at time $T^{j}(N)$ and ends at $T^{j}(N)+H^{j}(N)$; at time $T^{j}(N)$ the latter is a random variable, because of the second term $H^{j}(N)$. Obviously the same is true for the extra profit of the monopolist: if $r_{T}$ denotes his (expected) profit rate up to the first successful imitation at time $T^{j}(N)+H^{j}(N)$, bearing in mind (20) equation (19) at time $T^{j}(N)$ becomes

$$
\begin{aligned}
\frac{p_{k_{j}, N-1}}{w} & =\left(\frac{p_{j, N-1}}{w}-a_{j, N}\right) E\left(\int_{0}^{\min \left\{H^{j}(N), m\right\}} e^{-r_{T} \tau} d \tau\right)+ \\
& +\left(\frac{p_{j, N}}{w}-a_{j, N}\right) E\left(\int_{\min \left\{H^{j}(N), m\right\}}^{m} e^{-r \tau} d \tau\right)
\end{aligned}
$$

Theorem 11 The rate of profit $r_{T}^{j}$ of the monopolist in $\left[T^{j}(N), T^{j}(N)+H^{j}(N)\right]$ is an increasing function of the information content $S_{j}(N)$ of the $N$-th innovation.

Proof. Since we are assuming a Poisson random arrival of successful imitations with arrival rate $\mu, \operatorname{Pr}\left(H^{j}(N) \leq \tau\right)=1-e^{-\mu\left(S_{j}\right) \tau}$ and (23) becomes

$$
\begin{aligned}
& \frac{p_{k_{j}, N-1}}{w}=\left(\frac{p_{j, N-1}}{w}-a_{j, N}\right) \frac{1-e^{-\left(\mu\left(S_{j}(N)\right)+r_{T}\right) m}}{\mu\left(S_{j}(N)\right)+r_{T}}+ \\
& +\left(\frac{p_{j, N}}{w}-a_{j, N}\right)\left(\frac{1-e^{-r m}}{r}-\frac{1-e^{-\left(\mu\left(S_{j}(N)\right)+r\right) m}}{\mu\left(S_{j}(N)\right)+r}\right)
\end{aligned}
$$


As $p_{j, N-1}, p_{k_{j}, N-1}, p_{k_{j}, N}$ are given by (21) (22), $a_{j, N}$ by (17), and $r$ is fixed, equation (24) defines implicitly $r_{T}$ as a function of $\mu\left(S_{j}(N)\right)$ (and $\lambda_{j}$ ).

Troublesome, but elementary calculus show that

$$
\frac{\partial r_{T}}{\partial \mu\left(S_{j}\right)}<0
$$

Together with the fact that the arrival rate $\mu\left(S_{j}(N)\right)$ is a decreasing function of the information content $S_{j}(N)$ of the $N$-th innovation, (25) proves the theorem.

\section{Creative destruction and asymptotic behaviour}

By resorting to logarithms in (22), it is now possible to determine the long run price growth rates:

$$
\frac{\log p_{j, N_{t+\Delta}^{j}}-\log p_{j, N_{t}^{j}}}{\Delta}=-\frac{N_{t+\Delta}^{j} \frac{\sum_{n=1}^{N_{t+\Delta}^{j}} \lambda_{j}(n)}{N_{t+\Delta}^{j}}-N_{t}^{j} \frac{\sum_{n=1}^{N_{t}^{j}} \lambda_{j}(n)}{N_{t}^{j}}}{\Delta},
$$

Clearly, the growth rate depends both on the number $N_{t}^{j}$ of innovations introduced in sector $j$ and on the sequence of productivity growth rates $\left\{\lambda_{j}(n)\right\}_{n=1}^{N_{t}^{j}}$ generated by successive innovations. To estimate the expected value of (26) we have to introduce a further but quite natural assumption.

Axiom 12 The (random) arrival of an innovation wave and the (random) technological search $S_{z}$ which the $j$-th innovator locks into are independent variables.

Notice, therefore, that the expected value in (26)

$$
E\left(\frac{\sum_{n=1}^{N_{t}^{j}} \lambda_{j}(n)}{N_{t}^{j}}\right) \rightarrow \lambda^{*}=\sum_{z=1}^{Z} \lambda_{z} p_{z} \text { as } t \rightarrow+\infty
$$

by the law of large numbers, since $N_{t}^{j} \rightarrow+\infty$ as $t \rightarrow+\infty$. On the other hand, by the renewal theorem $E\left(N_{t}\right) \sim \frac{t}{\omega}$ and $E\left(N_{t}^{j}\right) \sim \frac{E\left(N_{t}^{j}\right)}{\alpha S^{*}}$ as $t \rightarrow+\infty$. Thus

$$
E\left(N_{t}^{j}\right) \sim \frac{t}{\omega \alpha S^{*}} \text { as } t \rightarrow+\infty
$$


Introducing (27) and (28) into (26), we derive the expected growth rate

$$
E\left(\frac{\log p_{j, N_{t+\Delta}^{j}}-\log p_{j, N_{t}^{j}}}{\Delta}\right) \sim \lambda^{*} \frac{1}{\omega \alpha S^{*}} \text { as } t \rightarrow+\infty .
$$

Analogously, it is quite straightforward to assess the medium run consequences of an innovation wave on the relative price $\frac{p_{j}}{p_{j^{\prime}}}$. Assume that both sectors are affected by an innovation wave in $(t, t+\Delta)$, then

$$
\log \frac{p_{j, N_{t+\Delta}^{j}}}{p_{j^{\prime}, N_{t+\Delta}^{j^{\prime}}}}-\log \frac{p_{j, N_{t}^{j}}}{p_{j^{\prime}, N_{t}^{j^{\prime}}}}=-\left(\lambda_{j}\left(N_{t+\Delta}^{j}\right)-\lambda_{j^{\prime}}\left(N_{t+\Delta}^{j^{\prime}}\right)\right)
$$

It is clear that, if the $j$-th innovator has locked in a longer search than the $j^{\prime}$-th, $\lambda_{j}\left(N_{t+\Delta}^{j}\right)-\lambda_{j^{\prime}}\left(N_{t+\Delta}^{j^{\prime}}\right)>0$, thus the relative price declines. This is all the more true if the $j^{\prime}$-th sector hasn't been at all affected by the innovation wave , because in this case the right hand side of (29) becomes simply $-\lambda_{j}\left(N_{t+\Delta}^{j}\right)$. This is only a medium run effect since it may either be reverted or reinforced by a successive innovation wave and it is, in any case, expected to average out (28) in the long run.

Equation (21) also allows to rank techniques by their profit rates at a given time $t$; the profit rate $r_{j, n}(t)$ of technique $n$ in sector $j$ at time $t$ is the unique positive solution, if any, of

$$
e^{-r_{j, n}} \frac{1-e^{-r_{j, n} m}}{r_{j, n}}=\frac{a_{k_{j}, n}}{\frac{p_{j}(t)}{w}-a_{j, n}}
$$

Since the function on the left hand side of (30) is decreasing in $r$ while that on the right hand side is increasing in $t$ and decreasing in $n, r_{j, n}(t)$ turns out to be decreasing in $t$ and increasing in $n$. The least $n$ for which there exists $r_{j, n}(t)>0$ is the least efficient technique still in use at time $t$, we denote this technique by $n_{t}^{j}$. As innovations occur though avalanches, old techniques become obsolete and the capital stock which embodies them scrapped costlessly. Thus, at each innovation wave, creative destruction takes place ${ }^{7}$.

We can rewrite (30) as follows

$$
e^{-r_{j, n_{t}^{j}}} \frac{1-e^{-m r_{j, n_{t}^{j}}}}{r_{j, n_{t}^{j}}}=\frac{a_{k_{j}, n_{t}^{j}}}{\left(a_{j, n_{t}^{j}}+\frac{a_{k_{j}, n_{t}^{j}}}{e^{-r \frac{1-e^{-r m}}{r}}}\right) e^{-\bar{\lambda}_{j}\left(N_{t}^{j}-n_{t}^{j}\right)}-a_{j, n_{t}^{j}}}
$$

\footnotetext{
${ }^{7}$ In $(30)$ we have assumed that there is a compatibility constraint on the verical integrated sector and the capital good necessary to produce the $j$-th commodity with the technique $n$ is produced by the same techinque on its part, even if at time $t$ more efficient technique are in use. It is possible to change this assumption and allowing the productuion of the old type capital good by the best technique in use at time $t$; this amounts to substitute the numerator of the right hand side of (30) by $a_{k_{j}, N_{t}^{j}}$. This cange does not affect the result.
} 
Where $\bar{\lambda}_{j}=\frac{1}{N_{t}^{j}-n_{t}^{j}} \sum_{n=n_{t}^{j}+1}^{N_{t}^{j}} \lambda_{j}(n)$.

Formula (31) can be interpreted as a trade off between medium run sector growth rate $\bar{\lambda}_{j}$ and the number $N_{t}^{j}-n_{t}^{j}$ of profitable techniques in use in sector $j$. The larger is the productivity rate achieved by the leader firm in the recent past, the smaller is the number of obsolete techniques sill capable of generating profit. In the medium run sectors with the fastest productivity growth experience also the strongest effects of creative destruction. On the other hand productivity growth rates are expected to converge to a common mean value, therefore sectors presently experiencing high levels of creative destruction may be expected to progressively register a slow down of this effect in the future.

In Figure 2 we show numerical simulations for the creative destruction pro$\operatorname{cess}^{8}$. The boxes represent the leading technology, while the triangles represent the least active one.
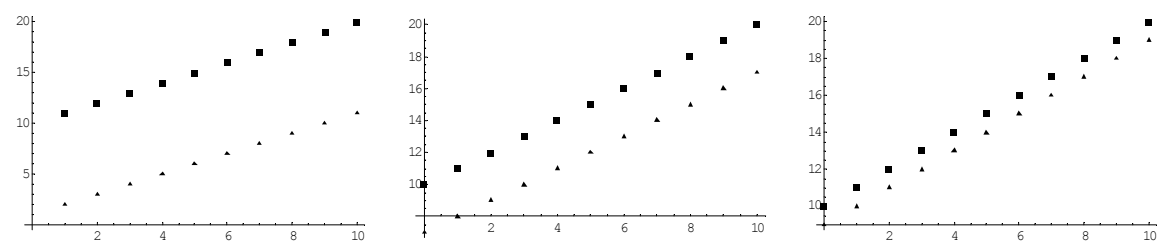

Figure 2: LHS $\bar{\lambda}_{j}=0.02$, middle $\bar{\lambda}_{j}=0.05$ and RHS $\bar{\lambda}_{j}=0.1$

\subsection{Structural comparisons}

It is now interesting to compare economies with different structural characteristics. If we call $\gamma_{I}$ the average long run increase in productivity for $t \rightarrow \infty$, it follows from the foregoing discussion that:

$$
\gamma_{I}=\frac{\lambda^{*}}{\alpha\left(S^{*}\right)^{2}} h t
$$

(32) shows that productivity growth depends crucially on the relation of $\lambda^{*}$ with $S^{*}$. This relation highlights the fact that $\frac{\lambda^{*}}{\left(S^{*}\right)^{2}}$ is an index assessing the return of an innovation, measured in terms of productivity gains, relative to the innovation costs, measured in terms of innovation difficulty. It is clear that more productive techniques, with a higher $\lambda$, needn't necessarily yield a higher long term productivity growth if the latter does not outweigh the implied informational cost. We may now use this result for a comparative purpose. It is

\footnotetext{
${ }^{8}$ We normalise $a_{j, 0}=a_{k_{j}, 0}=1$ and set $r=0.05$ and $m=2$.
} 
immediate to check that economies with a higher driving force $h$, other things being equal, exhibit a higher productivity and income growth in the long run. It is also straightforward that economies with a higher $\alpha$, that is with a higher threshold in terms of sector density for information to pass through, feature instead a lower growth rate. Consider, now, two economies $A$ and $B$, with a broadly similar knowledge base allowing them to explore and innovate on the grounds of two techniques having an informational sequence that is equal in both, $S_{1}^{A}=S_{1}^{B}=S_{1}$, the short sequence, and $S_{2}^{A}=S_{2}^{B}=S_{2}$, the long one, and subject to the same external driving force, $h$. The difference lies in a different sector distribution, $B$ possessing relatively more technologically advanced sectors than $A$. It follows that $p^{A}>>p^{B}$. Thus, the technologies introduced by country $A$ will, on average, be less technologically developed than those introduced by country $B$, i.e. $\lambda_{A}^{*}<<\lambda_{B}^{*}$. Further, we have that $S_{A}^{*}<<S_{B}^{*}$, and consequently the waiting time between new technologies will be lower in country $A$ than in country $B$, i.e. $\omega^{A}<<\omega^{B}$. This implies that $E\left(N_{t}^{A}\right) \sim \frac{t}{\omega^{A}}>>E\left(N_{t}^{B}\right) \sim \frac{t}{\omega^{B}}$. These structural features identify a frequency effect stating that country $A$ will introduce new technologies at a higher rate, the waiting time being lower, as well as a mass effect implying that its innovations are less developed and hence less productive than country $B$.

Putting these effects together we have that, in general, $\gamma_{I}^{A} \neq \gamma_{I}^{B}$. Whether the mass effect prevails over the frequency effect or vice versa depends, given (32) and for given $\lambda$ 's and $S$ 's, on the measure of probability $p$. Hence by differentiating $\gamma_{I}(p)$ with respect to $p$, it is easy to see that $\gamma_{I}(p)$ is an increasing function of $p$ if

$$
p<\frac{\left(\lambda_{1}-\lambda_{2}\right) S_{2}-2 \lambda_{2}\left(S_{1}-S_{2}\right)}{\left(S_{1}-S_{2}\right)\left(\lambda_{1}-\lambda_{2}\right)}
$$

It is clear that $\gamma_{I}(p)$ is an increasing function in $[0,1]$ if the right hand side of (33) is larger than 1 . This is true provided that

$$
\frac{\lambda_{2}-\lambda_{1}}{\lambda_{1}+\lambda_{2}}<\frac{S_{2}-S_{1}}{S_{2}}
$$

On the other hand, if the right hand side of (33) is negative, then $\gamma_{I}(p)$ is an decreasing function in $[0,1]$. This is true if

$$
\frac{\lambda_{2}-\lambda_{1}}{2 \lambda_{2}}>\frac{S_{2}-S_{1}}{S_{2}}
$$

Condition (35) can be met for $\lambda_{2}>\lambda_{1}>0$ only if $2 S_{1}>S_{2}$.

Conditions (34) and (35) show clearly that the prevalence of the mass effect is mainly due to the difference in productivity growth rates whilst the frequency effect owes to the difference in the length of informational sequences. 


\section{Conclusions}

It has been recently pointed out that innovation generates a process of technological convergence. This fact owes, on the one hand, on the searching efforts performed by firms and, on the other, to the spreading and spilling over of information which firms also determine. This paper investigates innovation and imitation as a searching and learning process organized by an information seeking routine. Firms are rationality bounded and heterogeneous as to their technological capabilities and, more generally, as to the cognitive tools they avail themselves of. This fact implies that the search process is localized in knowledge specific neighbourhoods made up by firms whose domain of activity, knowledge base and technologies are effectively understood and potentially apprehended and which define cognitive vicinity for each firm in the economy we study. It is through these neighbourhoods that information is relatively most likely to spread and that can be identified by means of a least entropy measure. Within such neighbourhoods firms' probability of gathering useful information, although different, can be taken as not too divergent, Wall Street brokers understanding each other as well as soy beans farmers do. This stylized fact enables us to study a dynamic process set in motion by an initial idiosyncratic innovative shock causing what in recent self organized criticality literature has been called an avalanche. The paper shows that its size depends positively on (i) the probability of information to be passed on from firm to firm and negatively on (ii) the number of information 'bits' to be collected before an innovation can actually be achieved. The pass-through probability is conjectured to be dependent on the externalities yielded by sector density reflecting the degree of specialization achieved by the economy. It is the spreading through different sectors of the initial innovative thrust that makes for technological convergence to occur. The waiting period between avalanches is shown to depend negatively, i.e. they occur more frequently, on the probability that an idiosyncratic shock is dealt the economy and positively, i.e. they are more far apart, on the number of informative 'bits'. These elements are structural features of an economy: the greater is the number of sectors the more the same knowledge base is shared and the more specialized is the economy: hence, the greater is the probability that information spills over. Although the paper deals with the initial shock as an exogenous occurrence, it is quite clear that it depends on the search efforts, the research and development expenditure, and more generally the technological capabilities available to the economy as a whole.

Imitation is the band wagon effect following innovation. Firms with a technique below the technology frontier observe the leaders and imitate on the bases of a Poisson arrival rate which depends on technological complexity as it is construed by the length of the information sequence required for the original innovation. Imitation then follows a logistic curve and since the probability of imitation is realistically assumed to be substantially higher than the probability of an avalanche occurring, firms do catch up so that the proportion of technologically top ranking firms tends to one and those of the lower ranks tend to zero. 
Innovation increases productivity and innovators earn quasi rents which are limited by the speed of imitation which, as it has been seen, is in turn dependent on the complexity of the original innovation. Prices and costs are determined basically by direct and indirect labour and it is to these inputs that productivity growth must be ascribed. Since the wage-profit distributive shares are shown to be remarkably steady for long periods of time, the long term profit rate is assumed to be constant letting all productivity gains accrue to the wage rate. As labour costs decrease so do relative prices causing lower than frontier techniques to become obsolete and be scrapped determining creative destruction in a Schumpeterian sense. The speed and intensity of this process hinges upon long term productivity growth which is shown to depend on structural parameters. The sector composition of an economy which is crucial to set the probability for innovators of locking into either long or short informational sequences leading to more or less productive technologies is shown to play an important role in the economy's long term performance. The waiting period theorem tells that more productive techniques are less frequent but have a greater mass effect. Historical evidence has indeed shown that more technologically advanced economies may, at times, grow less fast than less technologically advanced ones where innovations are, however, more frequent.

\section{References}

[1] Aghion P. and Howitt P. (1998): Endogenous Growth Theory, Massachusetts Institute of Technology.

[2] Aghion P. and Howitt P. (1992): A model of growth through creative destruction, Econometrica 60: 323 - 51.

[3] Arthur W. B. (1994): Increasing returns and path dependence in the economy, University of Michigan Press;

[4] Auerswald P.,Kauffman S.,Lobo J. and Shell K. (2000): The production recipes approach to modelling technological innovation: An application to learning by doing: Journal of Iconic Dynamics \& Control, 24, 389-450

[5] Bak P.,Tang C. and Wiesenfeld K. (1988): Self-organised criticality, Physical Review A: 38,364.

[6] Dosi G.(1988): Sources, procedures and macroeconomic effects of innovation, Journal of Economic Literature: 26,3 .

[7] Fai F. and von Tunzelmann N. (2001): Industry-specific competencies and converging technological systems: evidence from patents, Structural Change and Economic Dynamics, 12, p. 141-170.

[8] Feller W. (1971): An Introduction to Probability Theory and its Applications, Wiley Series in Probability and Mathematical Statistics, New York. 
[9] Franke R. (2001): Wave trains, innovation noise and long waves, Journal of Economic Behavior and Organization: 45, 49-68.

[10] Klir G.J. and Folger T. A. (1988): Fuzzy sets, uncertainty and information, Prentice Hall, London.

[11] Iwai K.(1984): Schumpeterian dynamics. Part II. Technological progress, firm growth and 'economic selection': Journal of Economic Behavior and Organization, 5, 321-351.

[12] Iwai K. (2000): A contribution to the evolutionary theory of innovation, imitation and growth. Journal of Economic Behavior and Organization, 43, 167-198.

[13] Nelson R. R. and Winter G.S. (1982): An evolutionary Theory of Economic Change, The Belknap Press, Cambridge, Mass.

[14] Pasinetti L. L.(1981): Structural Change and Economic Growth. Cambridge University Press, Cambridge.

[15] Pasinetti L. L.(1993): Structural Economic Dynamics. Cambridge University Press, Cambridge.

[16] Potts J. (2001): Knowledge and Markets. Journal of Evolutionary economics, 2,4 413-432.

[17] Romer P.M.(1990): Endogenous technical change. Journal of Political Economy, 98,5.

[18] Rosenberg, N. (1976): Perspectives on Technology, Cambridge University Press, Cambridge.

[19] Vespignani A., Zapperi S. (1998): How Self-organized criticality works: A unified mean-field picture, Physical Review E, vol. 57 n.6, p. 6345. 